\title{
Hyper-reoriented walking in minimal space
}

\author{
Sandro Ropelato ${ }^{1} \cdot$ Marino Menozzi $^{1} \cdot$ Melody Ying-Yu Huang ${ }^{1,2}$
}

Received: 11 November 2020 / Accepted: 15 November 2021 / Published online: 10 December 2021

(c) The Author(s) 2021

\begin{abstract}
We present a new reorientation technique, "hyper-reoriented walking," which greatly reduces the amount of physical space required in virtual reality (VR) applications asking participants to walk along a grid-like path (such as the most common layout in department stores). In hyper-reoriented walking, users walk along the gridlines with a virtual speed of twice the speed of real walking and perform turns at cross-points on the grid with half the speed of the rotation speed in the physical space. The impact of the technique on participants' sense of orientation and increase in simulator sickness was investigated experimentally involving 19 participants walking in a labyrinth of infinite size that included straight corridors and $90^{\circ}$ T-junctions at the end of the corridors. Walking accuracy was assessed by tracking the position of the head mounted display, and cyber-sickness was recorded with the simulator sickness questionnaire and with open questions. Walking straight forward was found to closely match the ideal path, which is the grid line, but slight errors occasionally occurred when participants turned at the T-junctions. A correction algorithm was therefore necessary to bring users back to the gridline. For VR experiments in a grid-like labyrinth with paths of $5 \mathrm{~m}$ in length, the technique reduces required size of the tracked physical walking area to $3 \mathrm{~m} \times 2 \mathrm{~m}$.
\end{abstract}

Keywords Virtual reality $\cdot$ Virtual locomotion $\cdot$ Natural walking $\cdot$ Redirected walking

\section{Introduction}

Exploring environments by moving in space is a common task in user studies, for example when studying customer behavior inside a store (Doniec et al. 2020). Similarly, usability evaluations of products may force participants to move in space, for instance when investigating the ergonomics of entering and exiting passenger vehicles (Lawson et al. 2015). There has been a trend toward performing user studies in virtual reality (VR) for various reasons, including to address safety concerns when research questions require confronting users with potentially dangerous situations (Shibata et al. 2002), time and cost-effectiveness in studies requiring a difficult and time-consuming setup (Mottura et al. 2003), or ethical issues when humans or animals are

Sandro Ropelato

sandro.ropelato@gmail.com

1 ETH Zurich, Human Factors Engineering, Zurich, Switzerland

2 Department of Anaesthesiology and Pain Medicine, Bern University Hospital (Inselspital), University of Bern, Bern, Switzerland involved (Balcombe 2004). Furthermore, environmental bias is better under control and study settings are fully replicable when user studies are implemented with VR technology (Miller 2016). In addition to studies, there is an increasing number of use cases where users explore virtual environments, such as gaming (Zyda 2005), rehabilitation (Meldrum et al. 2012), and education (Englund et al. 2017).

When exploring space in VR, there is a need for interfaces enabling participants to travel intuitively in virtual space. Various techniques have been developed to enable easy and efficient locomotion of users in virtual space, such as steering-based, selection-based, manipulation-based, and walking-based techniques (Al Zayer et al. 2020). The applicability of a specific locomotion technique varies depending on the nature of the study and on adverse effects on the participants of a study, which are introduced by the technique. Altering a user's virtual position or orientation without providing adequate proprioceptive and vestibular stimulation, as with the teleporting repositioning technique, may disrupt spatial updating (Cherep et al. 2020) and therefore limit the user's sense of orientation. Similarly, vestibular and proprioceptive information gained through locomotion by gesture, such as walking in place (Slater et al. 1995; Kim 
et al. 2021), differs from vestibular and proprioceptive information gained when walking in reality and will therefore negatively affect the feeling of presence (Usoh et al. 1999).

From a viewpoint of ecological validity, walking in VR should be mediated by a technique based on natural walking (see Cardoso et al. 2019 for an exhaustive survey on natural walking techniques in VR). Natural walking in VR works well for set-ups in which there is enough physical space available to include the simulated virtual space. In order to cope with spatial constraints, various walking techniques have been proposed and tested, such as repositioning techniques, locomotion using gestures, and redirected walking (Nilsson et al. 2018). As mentioned above, the first two techniques suffer from immense drawbacks such as limiting the user's sense of orientation or generating situations of conflicting vestibular and proprioceptive information.

In the beginning of this millennium, Razzaque et al. $(2001,2002)$ introduced the technique of redirected walking. Redirected walking relies on users' inability to notice small deviations in the virtual representation of the visual environment when walking (see Sun et al. 2020 for a summary of the state-of-art in redirection walking techniques). For example, Steinicke et al. $(2008,2010)$ discovered that users can be made to believe that they moved along a straight line when in fact they walked in a circle. For the illusion to work, the radius of the circle must be at least $22 \mathrm{~m}$, which requires a physical space with a floor area of $44 \mathrm{~m} \times 44 \mathrm{~m}$ or larger. For smaller floor areas, the illusion generates a noticeable discrepancy between physical and visual perception while walking on a long, straight virtual path. Reducing the discrepancy by limiting the effect of the illusion, as with redirected walking, would cause participants to bump into the boundaries of the physical space. This can be avoided applying various strategies, such as instructing the users to either perform a so-called freeze turn, where they physically turn but the visual orientation does not change, or a 2:1 turn, where they perform a $180^{\circ}$ turn but the visual representation indicates a full $360^{\circ}$ turn (Williams et al. 2007). With both methods, users are facing the same direction at the end of the turn as they did when they started turning, but their body has physically changed orientation and is heading away from the boundary of the physical space. Unfortunately, the benefit of the 2:1 turning technique is compensated by the costs of involving additional cognitive resources (Williams et al. 2007). Further, both turning techniques are problematic as users are forced to perform a task which not present in natural conditions. Consequently, negative effects may break the sense of presence of the user.

Negative effects of reorientation techniques based on turning are less pronounced if the turns are a natural component of the tasks in the virtual environment (Cardoso et al. 2019). For instance, turns in a store occur at the ends of aisles. Situations typically requiring $90^{\circ}$ turns at the ends of aisles are found in supermarkets and drugstores, where displays and aisles typically are arranged in a grid layout (Zentes et al. 2017). In such stores, $90^{\circ}$ turns are a normal part of the walking task and therefore provide favorable conditions for reorientation of the user in the VR environment by means of turning techniques. In order to maximize the reorientation effect and therefore minimize required dimensions of the physical room, users approaching the boundary of the physical space should be forced to physically turn by $180^{\circ}$ so they walk back along the path they used to come. As in the case of a virtual store with a grid layout, when users are at the end of the aisles they would perform a $180^{\circ}$ physical turn but experience a $90^{\circ}$ turn in the VR environment. A further room compression could be achieved by also applying a gain to the speed of walking. We propose a technique combining reorientation by turning and applying a gain to walking speed, and we called it "hyper-reoriented walking".

Virtual environments, which could benefit from hyperreoriented walking have been reported in the scientific literature. For instance, in consumer behavior research, gridlike virtual shops have been used to investigate the effect of abnormal looking or perishable fruits and vegetables on the perception and purchase behavior (Verhulst et al. 2017; Lombart et al. 2020), to investigate whether consumers standing in front of a shelf in a real supermarket take same decisions as consumers standing in front of a shelf in a virtual supermarket (Siegrist et al. 2019), and to inquire the consumer behavior when purchasing mass consumption products (Quevedo et al. 2018). Grid-like walking paths in a virtual environments have also been used in logistics research. Reif et al. (2008) used treadmill mediated walking through storage shelfs in a virtual warehouse to obtain performance data for planning manual order picking systems.

In this paper, we provide proof of concept for hyperreoriented walking in a severely limited space with a floor surface of $3 \mathrm{~m} \times 1 \mathrm{~m}$. In our experiment we record walking path, motion sickness, perceived orientation and walking distance in users exploring an infinitely large VR space. We used a labyrinth instead of a store with a square grid layout to better give the impression of an infinite space. The labyrinth consisted of corridors of equal lengths and ending in T-junctions requiring a $90^{\circ}$ turn.

\section{Related work}

A similar reorientation technique as the one we suggest here has been proposed by Yu et al. (2018). In the virtual environment proposed by Yu et al. (2018), users walk along tunnel segments. The segments are interconnected at their ends by $90^{\circ}$. At the end of each tunnel segment, users were required to seek a light switch to light up the next tunnel segment. While doing so, users were reoriented and physically rotated 
by $180^{\circ}$. The reorientation was achieved by adding $10 \%$ rotation speed to the absolute value of the rotation speed of the users head, thus added rotations were accumulated in the same direction. To achieve the accumulation of rotations, users were motivated to keep turning their head while searching for the light switch. The technique proposed by Yu et al. (2018) was implemented in a physical space of $4.6 \mathrm{~m} \times 1.5 \mathrm{~m}$. Their VR environment was experienced by dozens of visitors. A couple of impressions reported by the visitors are given but the setup of Yu et al. (2018) was not assessed by standardized metrics of cyber-sickness, presence etc.

A redirected walking technique reducing the amount of required physical space has been suggested by Langbehn et al. (2017). Langehn's method consists in applying bending gains (of the order between 2 and 4.35 in an example reported in the paper) on several curved physical paths and which are intersecting between them. At each intersection, users exploring Langbehn's virtual environment decide whether to turn left or right. Given the particular shape of the curved paths one of the turns is along the walking direction and the other forces the user to change the direction of walking. As the authors demonstrated (Langbehn et al. 2017) their technique enables to explore a $25 \mathrm{~m} \times 25 \mathrm{~m}$ virtual space within a $5 \mathrm{~m} \times 5 \mathrm{~m}$ physical setup.

An early paper investigating the viability of applying translational gains to overcome limitations of physical space in virtual environments was authored by Williams et al. (2006). In their walking experiment Williams et al. (2006) assessed the effect of different translational gains (1:1, 2:1, 10:1) on spatial localization memory. After a learning phase, in which users were asked to explore the virtual environment by walking and memorize the location of objects, participants were asked to close their eyes and to point to the object randomly selected by the experimenter. As memorization performance was not affected by variation in gain, Williams et al. (2006) concluded that applying translational gain is a feasible technique to use when exploring large virtual environments.

Steinicke et al. (2008) investigated the noticeability of rotational and translational gains by means of two separate psychophysical experiments. In a first experiment participants were asked to physically turn until they were stopped. At the end of the turn participants indicated whether they perceived a greater or smaller physical rotation than the visually stimulated rotation. Following their results, rotations of $68 \%$ more or $10 \%$ less than the perceived rotations are hardly noticeable. In the second experiment, participants were asked to walk a distance of $5 \mathrm{~m}$ in a virtual environment while the physical distance participants were required to walk varied between 3 and $7 \mathrm{~m}$. Results of the second experiment indicate that with translational gains between 0.78 and 1.22 virtual and physical translations are perceived the same in length. Steinicke et al. (2008) report average total SSQ scores for pre and post exposure. Averages were computed across all participants in the two studies and therefore do not allow to make a difference between the SSQ in the two studies.

\section{Methods}

In our work, we study the feasibility of applying a combination of the rotational and translational gain in separate sections of the virtual environment for compressing the required physical space. This so called hyper-reorientation technique was evaluated in terms of turning and walking accuracy as well as its effect on cyber-sickness. For the latter, the SSQ by Kennedy et al. (1993) was used.

\subsection{Participants}

Via mailing lists directed toward bachelor's and master's students at our university, we recruited a total of 21 participants (10 females) with an average [SD] age of 27.6 [4.7] years.

\subsection{Instrumentation}

We created a dynamically generated virtual labyrinth consisting of corridors and $90^{\circ}$ T-junctions with Unity 2018.3.7 (Unity Technologies, San Francisco, California, USA). The labyrinth was presented using an HTC Vive Pro (HTC Corporation, Taiwan) head-mounted display (HMD). With the HTC Vive pro it is possible to record the position and orientation of the HMD using two HTC Vive base stations placed at fixed locations in space. The accuracy of tracking the HTC Vive HMD has been shown to depend on whether the state of the HMD is static or dynamic (Borges et al. 2018). While the device is in static state, the tracking accuracy, expressed in standard deviations (SD) of repeated measures of position, is in the submillimeter range. Accuracy when the HMD is moving ranges from sub-millimeters to about $72 \mathrm{~mm}$, with a median SD of $2.2 \mathrm{~mm}$ (Borges et al. 2018). Position and orientation were tracked at a rate of $10 \mathrm{~Hz}$ and stored for further processing. After participants completed the turn at the T-junction and walked for $25 \mathrm{~cm}$ toward the next T-junction, the direction of turn (right or left) and the rotational error (difference between performed rotation and $180^{\circ}$ ) were computed and stored.

The experiment was run in a room with a size of $4.1 \mathrm{~m} \times 5.6 \mathrm{~m}$. A driving simulator and computers in the room limited the usable space for the simulation to $4.1 \mathrm{~m} \times 3.6 \mathrm{~m}$. After considering safety margins, a walkable area of $3 \mathrm{~m} \times 1 \mathrm{~m}$ was defined (Fig. 1).

In the VR labyrinth, participants walked along a corridor (Fig. 2, left) with a virtual length of $5 \mathrm{~m}$ before reaching the 


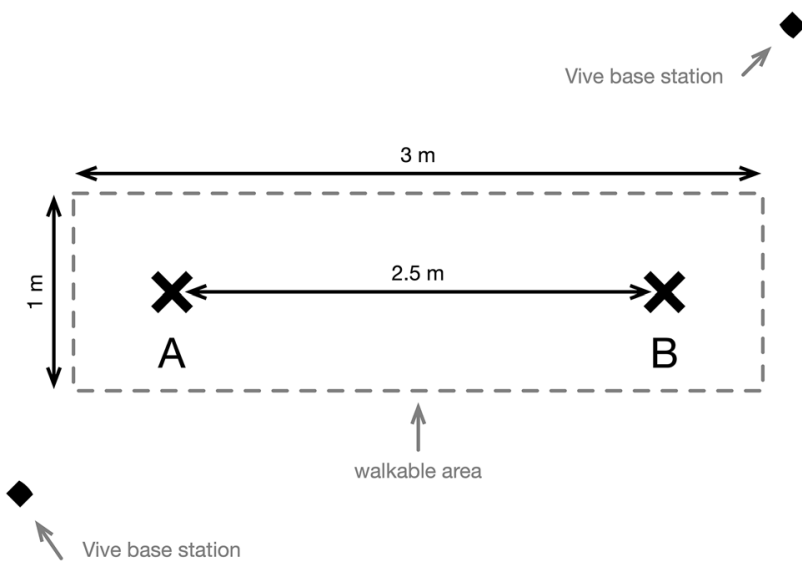

Fig. 1 Layout of the physical walking area, with turning points $A$ and $B$. Figure created with OmniGraffle 7.17 (The Omni Group, Seattle, Washington, USA)

next T-junction. The virtual width of the corridor was set to $0.7 \mathrm{~m}$. Figure 2 (right) shows a view of two corridors in the labyrinth, taken from the T-junction. The reorientation illusion was active in the play area starting $25 \mathrm{~cm}$ before the turning point and on the whole width of the play area $(1 \mathrm{~m})$. The illusion consisted in rotating the displayed labyrinth at half the rotation speed of the user in physical space. Therefore, the user was forced to perform a $180^{\circ}$ rotation in the physical space for a $90^{\circ}$ turn in VR. Once a turn was completed, the user walked along the next corridor. In some cases, users rotated too little or too much, causing a rotational error. Also, users could rotate at a different point than at the physical rotation points A or B placing them apart of the midline of the next corridor. A correction algorithm was applied in order to compensate for the rotational errors and the offset from the midline of the next corridor forcing the users to walk to the next physical turning point. The correction algorithm performed a weighted rotation of the labyrinth considering the misalignment angle between the midline of the virtual corridor and the orientation of the connection between the physical turning points A and B. The weighting factor was varied linearly with walking distance starting from 0 (no correction, starting $25 \mathrm{~cm}$ after the rotation) to 1 (total compensation of misalignment angle at $25 \mathrm{~cm}$ before the next physical turning point). Since the physical space was limited in walking direction, the virtual $5 \mathrm{~m}$ walking distance was realized by applying a gain of two to the longitudinal component of the virtual walking speed (parallel to line $\overline{A B}$ ). Figure 2 summarizes the settings applied during the various phases of the walking experiment. At each T-junction, participants were free to choose to go either right or left by rotating their body either clockwise or counter clockwise.

\subsection{Procedure}

Participants entered the lab and were free to look around. Before starting the experiment, participants were screened for susceptibility to develop simulator sickness, using the short version of the motion sickness susceptibility questionnaire (MSSQ-short), which includes 18 items (Golding 1998, 2006). To the best of our knowledge, the literature lacks a simple way to compute a threshold of the MSSQshort score preventing the development of moderate nausea. However, correlations between the MMSQ score for initiating moderate nausea and the time spent in simulation have been reported in the literature and summarized by Golding (1998). Summarized data refer to the long version of the MSSQ (MSSQ-long), which includes 54 items. Fortunately, Golding (1998) showed that the MMSQ-short can directly replace the MSSQ-long, and that MSSQ-short scores can be converted to MSSQ-long scores simply by multiplying MSSQ-short scores by 2.64 . Therefore, it is possible to use Golding's (1998) data about the correlation of the MSSQ-long score with exposure duration to determine the

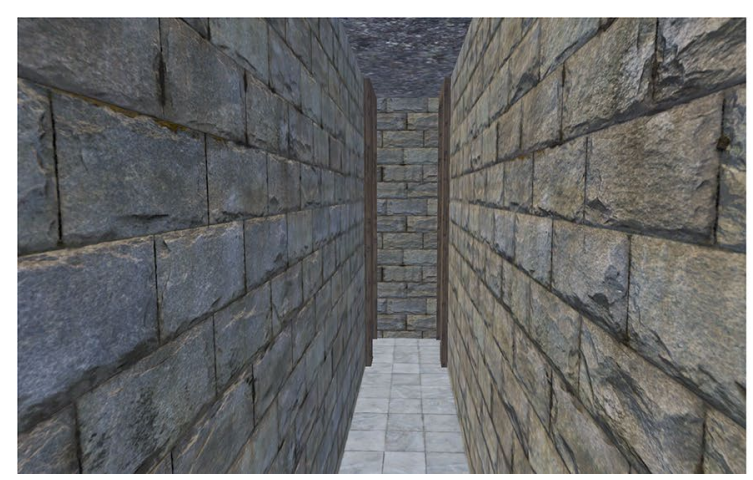

Fig. 2 View along a corridor (left) and from a T-junction (right) in the virtual labyrinth Figure created with Unity 2018.3.7 (Unity Technologies, San Francisco, California, USA), nVidia Shadowplay

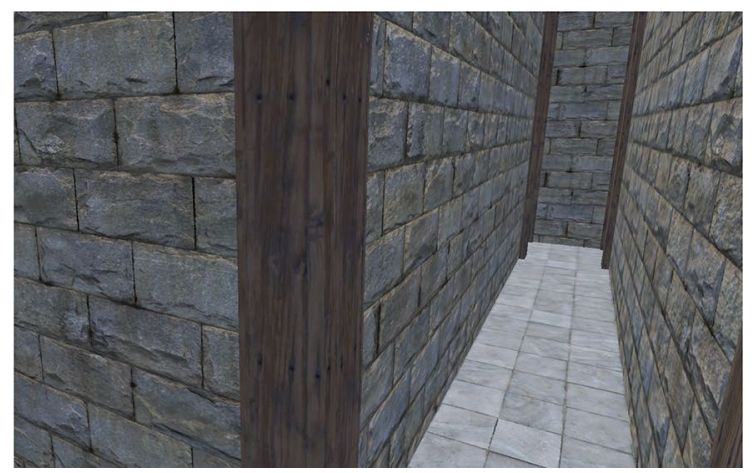

(Nvidia, Santa Clara, California, USA), and OmniGraffle 7.17 (The Omni Group, Seattle, Washington, USA) 
maximum exposure duration for preventing moderate nausea in VR when the MSSQ-short score is known.

In our experiment we used an exposure time of $5 \mathrm{~min}$ and retrieved the corresponding MSSQ-long score from Fig. 3 in Golding (1998), which is about 120. Then, from Fig. 1 in Golding (1998), we read that participants with a score of 120 belong to the 95 th percentile. From this, we estimated the MSSQ-short score using the mean $( \pm \mathrm{SD})$ of the MSSQshort score for a normative, young population (average age 26.0 years, $\mathrm{SD}=7.5$ years), which is $12.9 \pm 9.9$ (Golding, 2006). Considering that in a normal standard distribution the 95th percentile has a z-score of 1.65, the threshold MSSQshort score to consider for preventing moderate nausea after 5 min exposure is then $12.9+1.65 * 9.9 \approx 30$. A different way to compute the threshold for MSSQ-short is to divide the MSSQ-long threshold of 120 by 2.64 , which results in a MSSQ-short threshold value of 45 . In our screening, we used the more conservative value of 30 for MSSQ-short. The mean [SD] of the MSSQ-short score in the 21 participants was 10.06 [8.44]. One participant's MSSQ-short score exceeded 30 (MSSQ-short score 33.00) and a second participant with an MSSQ-short score of 26.25 aborted the study after showing strong cyber-sickness symptoms and did not complete the 15 turns. Both were excluded from the study and their was not used in the analysis.

The remaining 19 participants (average age of 28.1 [5.0] years, 8 females, average MSSQ-short score 8.11 [5.92]) filled in the simulator sickness questionnaire (SSQ; Kennedy et al. 1993), a first-time (baseline), before being equipped with the HMD. After participants felt comfortable with the HMD they were instructed to walk in the labyrinth until the T-junction at the end of the corridor

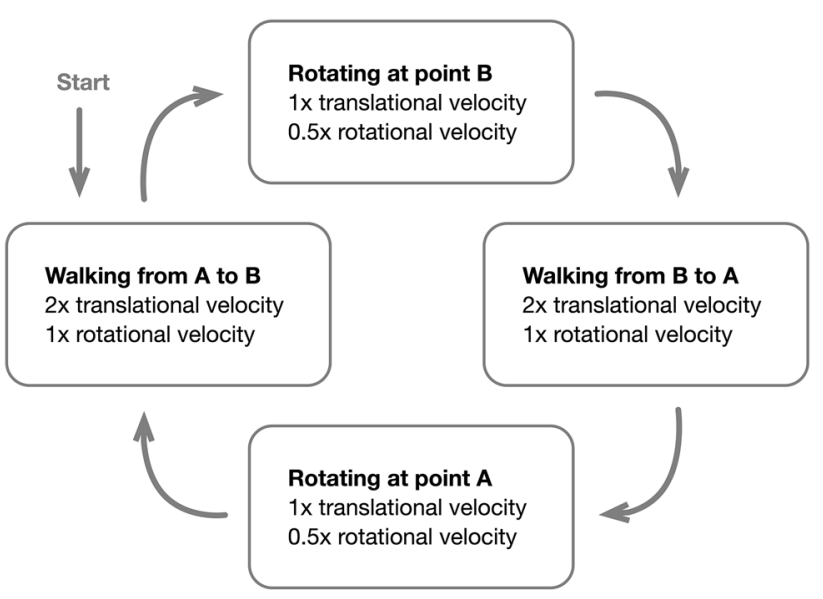

Fig. 3 The four states in which the participant moves through the labyrinth. When walking from point $\mathrm{A}$ to point $\mathrm{B}$, the translational velocity is doubled and the rotational velocity is normal. During a rotation at point $\mathrm{B}$, the translational velocity is normal and the rotational velocity is halved. Figure created with OmniGraffle 7.17 (The Omni Group, Seattle, Washington, USA) and then to turn either right or left, as they preferred, and walk to the next T-junction, and so forth. Participants were instructed to stop immediately if they felt discomfort or nausea. In order to keep the participants motivated throughout the task, they were informed that after a certain amount of time they would be asked a question. Participants then started walking through the VR labyrinth, and were stopped after completing 15 turns. After they had stopped, the experimenter asked them about the direction of their last turn in the labyrinth. Then, participants took off the HMD and answered the SSQ a second time. After completing the second SSQ, participants' subjective experience while walking in the labyrinth was collected with an open question asking: "Was there anything that felt strange or awkward while you were walking or turning? If so, please describe it in a few words." The magnitude of the reoriented walking illusion was assessed by asking the participants to estimate the path in meters in between the turns they walked in reality and the degrees they turned in reality when they were at the T-junction.

In order to prevent participants from accidentally bumping into walls or other objects, the experimenter followed them closely. To overcome disturbances caused by the HMD cable while walking, the experimenter held the cable at a position above the participant's head throughout the walking task. The entire experiment lasted approximately 20 min. The study protocol was approved by the ethics committee of the local university (Ethics Approval number EK 2019-N-140).

\subsection{Data analysis}

The walking accuracy, which is the agreement of the tracked path with the straight line connecting the turning points A and B (Fig. 1) was assessed by computing the root mean square (RMS) of the lateral deviations of the tracked path from the straight line. The participants' sense of orientation was assessed by analyzing the rotational error after completing a turn. For each participant, RMS values of rotational errors in all turns were computed and averaged across participants.

The increase in total SSQ score in in the scores of the sub-factors nausea, orientation, and dizziness (Kennedy et al. 1993) was analyzed by means of T-tests for dependent samples. Further on, a Pearson correlation was used to assess the correlation of the increase in SSQ scores (total and sub-factors) with the lateral deviation of the tracked path, the rotational error, exposure duration, and the MSSQ score. For the above-mentioned analysis, SPSS version 26 (IBM Corporation, Armonk, NY, USA) was used. 


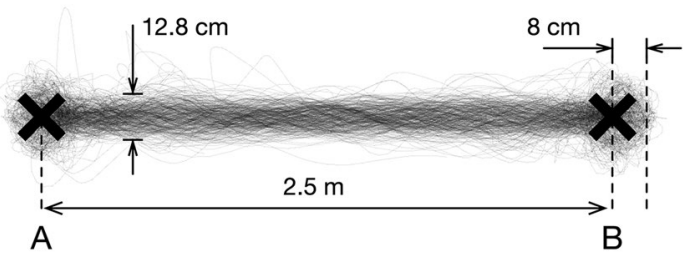

Fig. 4 Overlay of the head tracking paths of the 20 participants, recorded while walking in a labyrinth with a $2.5 \mathrm{~m}$ corridor ending at turning points $\mathrm{A}$ and $\mathrm{B}$. The envelope of overlaid tracking paths has a diameter of $12.8 \mathrm{~cm}$. At the turning points, tracked head positions in some participants overshoot the turning point by about $8 \mathrm{~cm}$. Figure created with OmniGraffle 7.17 (The Omni Group, Seattle, Washington, USA) and a custom program to visualize the walking paths written in Java 8 (Oracle Corporation, Redwood City, California, USA)

\section{Results}

In total the 19 participants completed 285 turns. On average they spent $251 \mathrm{~s}$ [64 s] in the VR simulation, with a minimum of $135 \mathrm{~s}$ and a maximum of $394 \mathrm{~s}$. From the start of the experiment until the first turn, the average duration was $27.3 \mathrm{~s}[10.0 \mathrm{~s}]$. The inter-turn duration for the rest of the turns varied from $4.7 \mathrm{~s}$ to $70.5 \mathrm{~s}$, with a $95 \%$ confidence interval between $5.5 \mathrm{~s}$ to $27.6 \mathrm{~s}$, and an average of $11.8 \mathrm{~s}$ [6.1 s]. Note that the reported times include the time participants needed to decide which way to turn.

Figure 4 represents an overlay of all paths walked by the 19 participants. Curves appear to fluctuate laterally in a sinusoidal-like manner along the straight connection of the turning points $\mathrm{A}$ and $\mathrm{B}$. A visual estimation reveals that turning at the turning points may cause a longitudinal overshoot of up to about $8 \mathrm{~cm}$.

The agreement between the tracked path and the straight line, was quantified by computing the root mean square (RMS) of the lateral deviations of the tracked path from the straight line. On average, the tracked paths of the 19 participants deviated laterally by $4.6 \mathrm{~cm}[1.5 \mathrm{~cm}]$ from the straight line.

After each turn, a rotational error was computed, indicating the error in heading to the next turning point. For each participant an average RMS value was computed, taking into account all rotational errors. The average of average RMS rotational errors across the 19 participants was $3.5^{\circ}\left[1.6^{\circ}\right]$.

Figure 5 reports averages and standard deviations for the total SSQ score and for the three SSQ sub-factors nausea, orientation and dizziness for the pre (white bars) and for the post (grey bars) recording of the SSQ. After 15 turns in the labyrinth (average walking time of $251 \mathrm{~s}$ [64 s]), the total SSQ score rose by 11.3 points and the increase was found to be significant (paired T-test, two sided, $\mathrm{T}(18)=4.422, p<0.001$ ). Also the scores in the three sub-factors increased after walking in the labyrinth $(+19.1$ for nausea, +9.6 for orientation,+30.0 for

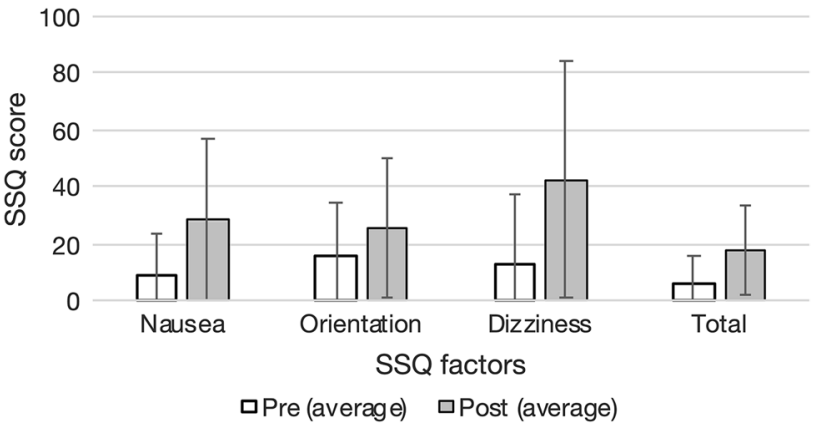

Fig. 5 Total SSQ scores and scores for the three sub-factors nausea, orientation, and dizziness collected before (pre) and after (post) exposure to the labyrinth task based on hyper-reoriented-walking. Figure created with Excel version 16.40 (Microsoft Corporation, Redmond, Washington, USA)

dizziness) and the increase was found to be significant in all three sub-factors $(\mathrm{T}(18)>3.314, p<0.005$ in all three comparisons). A Person correlation was used to analyze the interactions between the increases (differences in scores after and before the experiment) in the four SSQ scores, the MSSQ score, the exposure duration, the RMS deviation of the path from the straight line, and the RMS error in turns. The increase in total SSQ score (Pearson, two sided, $\mathrm{r}(17)=0.493, p<0.05)$ and the increase in the sub-factor orientation $(\mathrm{r}(17)=0.481, p<0.05)$ were found to be positively correlated to the MSSQ score. Therefore, the increase in SSQ total score and in the sub-factor orientation is more pronounced in participants with a higher MSSQ score than in those with a lower MSSQ score. The total path length and the exposure duration turned out to be correlated (Pearson, two sided, $\mathrm{r}(17)=-0.624, p<0.005$ ). No significant correlations were found between the four SSQ scores and the duration, the RMS deviation of the path from the straight line, and the RMS error in turns.

In subjective estimations of walking parameters, 11 participants $(58 \%)$ reported that they perceived the physical rotation to be less than $180^{\circ}$. Only $6(32 \%)$, believed that they were walking further than the $2.5 \mathrm{~m}$ they moved in reality.

From the participants' verbal feedback we learned that the turning felt "tough", "unstable", or "wobbly" in the beginning, but that they managed to adapt within the first few turns, so that walking on a straight line generally caused no problems. The initial steps after a turn, when the labyrinth forces the participants back onto the straight line to correct for the rotational error and the offset from the midline, were perceived as more difficult. Here also, participants managed to get used to the behavior. After the experiment, one participant reported having felt claustrophobic due to the narrow corridors $(70 \mathrm{~cm})$ in the simulation. 


\section{Discussion}

The present study aimed to show the proof of concept for a new redirection technique specifically designed for applications of virtual reality requiring walking and performed in a physical space very limited in size.

How well users are able to navigate through the virtual world was assessed on the basis of a user experiment in which participants walked in an infinite labyrinth consisting of corridors ending in $90^{\circ} \mathrm{T}$-junctions. The evaluation consisted of assessing how closely participants followed the ideal path between two turning points, turning accuracy, simulator sickness, and subjective estimates of experienced exposure to walking in the VR labyrinth. Our results revealed a lateral average deviation of the tracked path from the required straight path of $4.6 \mathrm{~cm}[1.5 \mathrm{~cm}]$ (average of individual RMS averages).

Lateral deviations when walking on a straight path occur naturally. Courtine et al. (2003) found an average deviation of the center of mass from the required straight path of $6 \mathrm{~cm}[1.6 \mathrm{~cm}]$. Although some error in tracking the HMD may occur due to a lack in precision of the HMD tracking (Borges et al. 2018), tracking the HMD seems to be in good agreement with tracking the center of mass of the body, the latter being a cardinal point in gait analysis studies.

Turning at the T-junction was associated with an average rotational error (average of individual RMS averages) of $3.5^{\circ}\left[1.6^{\circ}\right]$. After walking a $2.5 \mathrm{~m}$ long corridor, a rotation error of $3.5^{\circ}$ would cause the participant to arrive about $15 \mathrm{~cm}$ laterally away from the envisaged turning point at the other end of the corridor. Assuming the turning errors accumulate at each turn, a path offset of $2.3 \mathrm{~m}$ would appear after 15 turns. Under these conditions the lateral dimension of the walking area should be increased, e.g., to $2.3 \mathrm{~m}$, in order to avoid users bumping into the side wall. With a walking area of $3.3 \mathrm{~m} \times 3 \mathrm{~m}$ it would be possible to attenuate or eliminate the need to introduce a correction mechanism, which in some users caused discomfort. Attenuation or elimination of the correction mechanism could therefore reduce the simulator sickness.

Results plotted in Fig. 5 reveal an increase in SSQ scores after walking in the labyrinth. The effect of rotational gains on cyber-sickness (SSQ) was investigated in the studies by Schmitz et al. (2018) and Hildenbrandt et al. (2018). In both studies, participants were asked to walk and collect items in a virtual living room while rotational gains were applied in both studies. Gains were varied dynamically and either gradually increased or decreased in steps of 0.03 starting from a gain of 1.0 until participants noticed a breakdown in immersion after which a trial was terminated. In the study by Schmitz et al. (2018), average rotational gains were 0.58 for the decreasing and 1.85 for the increasing change of the gain. Based on the description of their experiment, this translates to an average of 21.16 turns each participant performed. Schmitz et al. reported an average SSQ total score of 15.28 before and 48.92 after exposure to the task. Hildenbrandt et al. (2018) found a similar pre to post variation in the average total SSQ score (averages pre total SSQ $=16.18$, post $=50.05$ ) with probably a similar range of adopted gains as in the experiment of Schmitz et al. (2018). The findings of the two studies support our findings as the application of rotational gains increases the total SSQ score and the level of the post SSQ score is about three times the level of the pre SSQ score. Also, our pre score level corresponds to the score level of the baseline measurements in Schmitzs' report, in which the task was run with a unity rotational gain with a pre score of 7.48 and a post score of 8.31. The pre SSQ scores in our study are comparable to Schmitz' baseline scores. However, the levels of the post SSQ scores in our study are lower than in Schmitz' and Hildenbrandt's studies. Among possible factors explaining found differences are differences in visual complexity of used test environments and the application of a dynamical varying rotational gain. Our virtual environment (Fig. 2) is simpler as compared to the living room used in the experiments of Schmitz and Hildenbrand. As shown by Jaeger et al. (2001) in a treadmill walking experiment without any redirection techniques applied, an environment with a lower level of visual details was found to produce a lower SSQ score than the environment with a higher level of details. Findings by Jaeger et al. (2001) reveal pre exposure SSQ total scores of 5.11 and 4.74 for the simple and the complex setting, respectively. SSQ total scores increased after exposure to 16.21 for the simple and to 20.07 for the complex environment, which is about a factor of 3 to 4 as compared to the pre exposure levels.

In agreement with others (Luebeck et al. 2015, Benzeroual et al. 2013), we found a positive and significant correlation between the MSSQ and the SSQ score. It is therefore possible that the MSSQ score in the population studied in Schmitz' and Hildenbrandt's is higher than in our population explaining the higher SSQ scores in their two papers.

The relatively low simulator sickness score shows that the new technique of hyper-reoriented walking is a viable locomotion technique in VR. It has to be noted, however, that the actual setup can only be applied in scenarios where participants walk through a labyrinth-like environment with a $90^{\circ}$ turn (either left or right) at the end of each corridor (i.e., no four-way intersections). Further, the distance between all intersections should be constant in order to avoid programming a logic to control for the sequence of corridor lengths, which is required for keeping the users within the physical boundaries. Virtual turns with other angles could be implemented with hyper-oriented walking, 
in analogy to what has been done by Langbehn et al. (2018) in their setup with bent paths. Other angles would require adjusting rotational gains in order to force the user to walk back and forth on the same line. For instance for a virtual turn of $120^{\circ}$ a gain of 1.5 and for a turn of $45^{\circ}$ a gain of 4 is necessary. By using different gains at different turning points, also other than grid-like paths could be implemented. However, we may assume that varying gains at each turning point could irritate the user and make it harder to adapt to the illusion.

While only four participants (20\%) believed they performed a $90^{\circ}$ turn, we were surprised that the illusion of rotation worked so well that more than half of the participants could be tricked into thinking they turned substantially less than $180^{\circ}$. Given that all participants were familiar with the physical dimensions of the room, it is not surprising that the majority (55\%) correctly estimated the physical distance they walked. Only two people (10\%) believed the illusion that walked a distance of $5 \mathrm{~m}$ between the turns.

\section{Conclusion}

From our findings, we conclude that, within the scope of the limitations mentioned, our modified version of redirected walking is a practicable solution for implementing virtual environments in which users navigate by a walking technique performed in the real physical space. In our study, participants were able to quickly adapt to the system and navigate through the virtual scene. To understand how much the size of our relatively small lab in which the experiment was conducted affected the participants' perception of the unnatural rotational gain, it would be interesting to run the same experiment in a larger room (e.g., a gymnasium) where the physical space is far larger than the simulated one.

In future work, we plan to investigate the effect of omission of the correction mechanism on required play area for a safe simulation.

Acknowledgements We thank Jeannie Wurz (Bern University Hospital) for her assistance with writing of this paper and for her comments that greatly improved the manuscript.

Funding Open access funding provided by Swiss Federal Institute of Technology Zurich. This research is funded by the EMDO Stiftung Zürich, 2018. Project title: Left or Right? Spontaneous Alternation Behavior in Zebrafish and Man (Gesuch Nr. 942) and ETH Zurich.

Data availability The data was generated at ETH Zurich. Derived data supporting the findings of this study are available from the corresponding author $[\mathrm{SR}]$ on request.

Code availability The source code is available from the corresponding author [SR] on request.

\section{Declarations}

Conflict of interest Sandro Ropelato, Marino Menozzi, and Melody Ying-Yu Huang declare that they have no conflict of interest.

Open Access This article is licensed under a Creative Commons Attribution 4.0 International License, which permits use, sharing, adaptation, distribution and reproduction in any medium or format, as long as you give appropriate credit to the original author(s) and the source, provide a link to the Creative Commons licence, and indicate if changes were made. The images or other third party material in this article are included in the article's Creative Commons licence, unless indicated otherwise in a credit line to the material. If material is not included in the article's Creative Commons licence and your intended use is not permitted by statutory regulation or exceeds the permitted use, you will need to obtain permission directly from the copyright holder. To view a copy of this licence, visit http://creativecommons.org/licenses/by/4.0/.

\section{References}

All Zayer M, MacNeilage P, Folmer E (2020) Virtual locomotion: a survey. IEEE Trans vis Comput Graph 26(6):2315-2334. https:// doi.org/10.1109/TVCG.2018.2887379

Balcombe J (2004) Medical training using simulation: toward fewer animals and safer patients. ATLA Altern Lab Anim 32(Suppl 1B):553-560. https://doi.org/10.1177/026119290403201s90

Benzeroual K, Allison RS (2013) Cyber (motion) sickness in active stereoscopic 3D gaming. In: Proceedings of the 2013 international conference on 3D imaging (IC3D), 1-7. https://doi.org/10.1109/ ic3d.2013.6732090

Borges M, Symington A, Coltin B, Smith T, Ventura R (2018) HTC Vive Analysis and accuracy improvement. In: IEEE/RSJ international conference on intelligent robots and systems (IROS) 2018:2610-2615. https://doi.org/10.1109/IROS.2018.8593707

Cardoso JCS, Perrotta A (2019) A survey of locomotion techniques for immersive VR applications on HMD. Comput Graph 85:55-73. https://doi.org/10.1016/j.cag.2019.09.005

Cherep LA, Lim AF, Kelly JW et al (2020) Spatial cognitive implications of teleporting through virtual environments. J Exp Psychol Appl 26(3):480-492. https://doi.org/10.1037/xap0000263

Courtine G, Schieppati M (2003) Human walking along a curved path. I Body trajectory, segment orientation and the effect of vision. Eur J Neurosci 18:177-190. https://doi.org/10.1046/j.1460-9568. 2003.02736.x

Doniec A, Lecoeuche S, Mandiau R, Sylvain A (2020) Purchase intention-based agent for customer behaviours. Inf Sci 521:380-397. https://doi.org/10.1016/j.ins.2020.02.054

Englund C, Olofsson AD, Price L (2017) Teaching with technology in higher education: understanding conceptual change and development in practice. High Educ Res Dev 35(1):73-87. https://doi.org/ 10.1080/07294360.2016.1171300

Golding JF (2006) Predicting individual differences in motion sickness susceptibility by questionnaire. Pers Individ Differ 41(2):237-248. https://doi.org/10.1016/j.paid.2006.01.012

Golding JF (1998) Motion sickness susceptibility questionnaire revised and its relationship to other forms of sickness. Brain Res Bull 47(5):507-516. https://doi.org/10.1016/S0361-9230(98)00091-4

Hildebrandt J, Schmitz P, Valdez AC, Kobbelt L, Ziefle M (2018) Get well soon! Human Factors influence on cybersickness after redirected walking exposure in virtual reality. In: Virtual, augmented and mixed reality: interaction, navigation, visualization, 
embodiment, and simulation, Vamr 2018, Pt I, 82-101. https:// doi.org/10.1007/978-3-319-91581-4_7

Jaeger BK, Mourant RR (2001) Comparison of simulator sickness using static and dynamic walking simulators. Proc Hum Factors Ergon Soc Annu Meet 45(27):1896-1900. https://doi.org/10.1177/ 154193120104502709

Kennedy RS, Lane NE, Berbaum KS, Lilienthal MG (1993) Simulator sickness questionnaire: an enhanced method for quantifying simulator sickness. Int J Aviat Psychol 3(3):203-220. https://doi. org/10.1207/s15327108ijap0303_3

Kim W, Xiong S (2021) User defined walking-in-place gestures for VR locomotion. Int J Hum Comput Stud 152:102648. https://doi.org/ 10.1016/j.ijhcs.2021.102648

Langbehn E, Lubos P, Bruder G, Steinicke F (2017) Application of redirected walking in room-scale VR. In: 2017 IEEE virtual reality (VR) Book Series:| Proceedings of the IEEE virtual reality annual international symposium 449-450. https://doi.org/10.1109/ VR.2017.7892373

Lawson G, Herriotts P, Malcom L, Gabrecht K, Hermawati S (2015) The use of virtual reality and physical tools in the development and validation of ease of use of entry and exit in passenger vehicles. Appl Ergon 48:240-251. https://doi.org/10.1016/j.apergo. 2014.12.007

Lombart C, Millan E, Normand JM, Verhulst A, Labbé-Pinlon B, Moreau G (2020) Effects of physical non-immersive virtual, and immersive virtual store environments on consumers' perceptions and purchase behavior. Comput Hum Behav. https://doi.org/10. 1016/j.chb.2020.106374

Luebeck AJA, Bos JE, Stins JF (2015) Motion in images is essential to cause motion sickness symptoms, but not to increase postural sway. Displays 38:55-61. https://doi.org/10.1016/j.displa.2015. 03.001

Meldrum D, Glennon A, Herman S, Murray D, McConn-Walsh R (2012) Virtual reality rehabilitation of balance: assessment of the usability of the Nintendo Wii® Fit Plus. Disabil Rehabil Assist Technol 7(3):205-210. https://doi.org/10.3109/17483107.2011. 616922

Miller HL, Bugnariu NL (2016) Level of immersion in virtual environments impacts the ability to assess and teach social skills in autism spectrum disorder. Cyberpsychol Behav Soc Netw 19(4):246-256. https://doi.org/10.1089/cyber.2014.0682

Mottura S, Viganò GP, Sacco M (2003) Virtual reality for product layout configuration. In: 1st international conference on research in virtual and rapid prototyping (VRAP)

Nilsson NC, Serafin S, Steinicke F, Nordahl R (2018) Natural walking in the virtual reality: a review. Comput Entertain 16(2):1-22. https://doi.org/10.1145/3180658

Quevedo WX, Venegas PF, Lopez VB, Gallardo CM, Acosta AG, Tapia JC, Andaluz VH (2018) Sales maximization based on neuromarketing techniques in virtual environments. In: Paolis LTD, Bourdot P (eds) Augmented Reality Virtual Reality and Computer Graphics AVR 2018 PT II. Springer. https://doi.org/10.1007/9783-319-95282-6_13

Razzaque S, Kohn Z, Whitton MC (2001) Redirected walking: Proceedings of Eurographics, 2001:105-106. https://doi.org/10.2312/ egs.20011036

Razzaque S, Swapp D, Slater M, Whitton MC, Steed A, (2002) Redirected walking in place. In: Proceedings of the workshop on virtual environments 2002:123-130

Reif R, Walch D (2008) Augmented and virtual reality applications in the field of logistics. Vis Comput 42(11):987-994. https://doi.org/ 10.1007/s00371-008-0271-7

Schmitz P, Hildenbrandt J, Valdez AC, Kobbelt L, Ziefle M (2018) You spin my head right round: threshold of limited immersion for rotation gains in redirected walking. IEEE Trans vis Util Comput Graph 24(4):1623-1632. https://doi.org/10.1109/TVCG.2018. 2793671

Shibata T, Fujihara H (2002) Development of railway VR safety simulation system. Q Rep RTRI 43(2):87-89. https://doi.org/10.2219/ rtriqr.43.87

Siegrist M, Ung CY, Zank M, Marinello M, Kunz A, Hartmann C, Menozzi M (2019) Consumers' food selection behaviors in threedimensional (3D) virtual reality. Food Res Int 117:50-59. https:// doi.org/10.1016/j.foodres.2018.02.033

Slater M, Usoh M, Steed A (1995) Taking steps: the influence of a walking technique on presence in virtual reality. ACM Trans Comput Hum Interact 2(3):201-219. https://doi.org/10.1145/ 210079.210084

Steinicke F, Bruder G, Jerald J, Frenz H, Lappe M (2008) Analysis of human sensitivity to redirected walking. In: Proceedings of the 15th ACM symposium on virtual reality software and technology. ACM, New York, 149-156

Steinicke F, Bruder G, Jerald J, Frenz H, Lappe M (2010) Estimation of detection thresholds for redirected walking techniques. IEEE Trans vis Util Comput Graph 16(1):17-27. https://doi.org/10. 1109/TVCG.2009.62

Sun Q, Patney A, Steinicke F (2020) Redirected walking. In: Magnor M, Sorkine-Hornung A (eds) Real VR - immersive digital reality. Springer, Switzerland, pp 285-292

Usoh M, Arthur K, Whitton MC et al. (1999) Walking > Walking-inPlace > Flying, in Virtual Environments. In: SIGGRAPH '99: Proceedings of the 26th annual conference on Computer graphics and interactive techniques, 359-364. https://doi.org/10.1145/ 311535.311589

Verhulst A, Lombart C, Normand, JM, Moreau G (2017) A study on the use of an immersive virtual reality store to investigate consumer perceptions and purchase behavior towards non-standard fruits and vegetables. In: 2017 IEEE annual symposium virtual reality (VR), https://doi.org/10.1109/VR.2017.7892231

Williams BW, Narasimham G, Rieser JJ (2006) Updating orientation in large virtual environments using scaled translational gain. In: Proceedings of the 3rd symposium on Applied perception in graphics and visualization, APGV '06, 21-28. https://doi.org/10. 1145/1140491.1140495

Williams B, Narasimham G, Rump B et al. (2007) Exploring large virtual environments with an HMD when physical space is limited. In: APGV '07: proceedings of the 4th symposium on Applied perception in graphics and visualization 2007:41-48. https://doi. org/10.1145/1272582.1272590

Yu R, Duer Z, Ogle T, Bowman DA, Tucker T, Hicks D, Choi D, Bush Z, Ngo H, Nguyen P, Liu X (2018) Experiencing an invisible world war I battlefield through narrative-driven redirected walking in virtual reality. In: 2018 IEEE conference on virtual reality and 3D user interfaces (VR). 313-19. https://doi.org/10.1109/VR. 2018.8448288

Zentes J, Morschett D, Schramm-Klein H (2017) Strategic retail management, 3rd edn. Springer Gabler. https://doi.org/10.1007/ 978-3-658-10183-1

Zyda M (2005) From visual simulation to virtual reality to games. Computer 38(9):25-32. https://doi.org/10.1109/MC.2005.297

Publisher's Note Springer Nature remains neutral with regard to jurisdictional claims in published maps and institutional affiliations. 